\title{
Urgences
}

\section{Discours de réception à l'Académie canadienne-française}

\section{Madeleine Gagnon}

Numéro 19, janvier 1988

Le tour du texte

URI : https://id.erudit.org/iderudit/025453ar

DOI : https://doi.org/10.7202/025453ar

Aller au sommaire du numéro

Éditeur(s)

Urgences

ISSN

0226-9554 (imprimé)

1927-3924 (numérique)

Découvrir la revue

Citer ce document

Gagnon, M. (1988). Discours de réception à l'Académie canadienne-française.

Urgences, (19), 103-106. https://doi.org/10.7202/025453ar d'utilisation que vous pouvez consulter en ligne.

https://apropos.erudit.org/fr/usagers/politique-dutilisation/ 


\section{Madeleine Gagnon DISCOURS DE RÉCEPTION À L'ACADÉMIE CANADIENNE-FRANÇAISE}

Le 16 octobre 1987. Madeleine Gagnon et Marcel Dubé furent reçus officiellement à l'Académie canadienne-française. Les discours de présentation furent faits par Fernande Saint-Martin dans le cas de Madeleine Gagnon, par Jean-Pierre Duquette dans celui de Marcel Dubé. Ce dernier el Madeleine Gagnon avaient été élus à l'Académie en juin. Ndlr

Monsieur le Président et distingués membres de l'Académie canadienne-française

Chers parents et amis

Me voici donc à l'Académie. Entre le moment où je reçus laimable invitation à me joindre à cette Académie et celui où ma candidature fut soumise aux voix de ses membres et agréée, un film se déroula dans mon esprit dont jaimerais vous communiquer les principales séquences. Mais d'abord,jaimerais remercier mes nouveaux collègues qui mont ainsi témoigné leur confiance et qui me font thonneur de maccueillir parmi eux. À ce sujet, permettez-moi de vous citer Paul Valéry, lors de son discours de Remerciement à l'Académie française: "Sur le seuil de votre audience, éprouvant invinciblement ce que l'on doit à votre faveur, on éprouve ce que lion est et lion se dit que tout arrive.»

La place dhonneur que vous maccordez, convoitée par certains alors que d'autres la craindraient, elle vient rajouter, surce métier décriture, choisi par ferveur et par amour uniquement, une valeur qui, elle, est toute morale; dune part, elle me dit la reconnaissance et l'estime de certains et d'autre part, par l'espèce de gloire qui elle procure, elle se trouve à donner aussi ce sentiment de dignité et de fierté d'un métier pas si mal accompli mais surtout, par dautres, estimé et valorisé.

Pour recevoir avec grâce de tels signes liés à la consécration, je veux dire pour que la suite de loeuvre, son perfectionnement et son approfondissement n'en souffrent pas, il faut, je crois, beaucoup d'humilité, cest-à-dire un véritable détachement de tout ce qui peut conduire au pouvoir et le générer. Depuis longtemps, je dirais même depuis les commencements de mon apprentissage 
dans l'écriture.jai choisi, pour des raisons dontje me suis souvent expliquée ailleurs et que le temps bref ne me permet pas de rappeler, jai opté pour ce que Maurice Blanchot, après Antonin Artaud, a su si bien nommer et pratiquer et qui se dit IMPOUNOIR.

En peu de mots, quill me soit permis de traduire ce que je pense de limpouvoir. Sans vouloir couper les mots en quatre, disons tout de même quil ne sagit pas d'un contre-pouvoir ou d'un autre pouvoir, sorte de bon pouvoir qui serait subversif. marginal et parallèle au mauvais pouvoir. Il sagit tout simplement d'une absence, comme diun vide intérieur, dune disponibilité qui laisserait très grande la place du désir, pour soi, pour les siens et pour sa propre littérature, de l'accueil des marques d'amitié, destime et de reconnaissance par lesquelles un individu ou un groupe atteint plus réellement, plus concrètement je dirais, une existence; le désir de ces marques qui sont vécues comme des preuves dexistence, le travail, même, pour qu'elles adviennent sont à la mesure du refus tout aussi ardent pour ce qui, vers l'individu ou le groupe, agit en sens contraire; refus de tout pouvoir. car, contrairement à diverses croyances courantes, il nien est pas de bon; refus de toute coercition comme de toutes formes dexclusion ou dassujettissement. ce qui signifie que pour moi la reconnaissance actuelle de l'Académie est partie intégrante de cette lutte contre tout pouvoir qui refúse le droit à l'existence de cela qui doit être reconnu.

On le voit bien, nous, du Québec et des autres minorités francophones nord-américaines, pour ne parler que de celles-ci, on comprend de mieux en mieux ce quil en est des divers refus dexistence et de reconnaissance des littératures minoritaires, de leurs écritures et de leurs paroles spécifiques, de la force de certaines d'entre elles. Je ne citerai pas de noms, il y en aurait trop et certains de ces noms me sont très chers et certaines de ces oeuvres me seraient très douces à nommer. Je dois à plusieurs dentre eux et plusieurs dentre elles une bonne part des raisons de ma présence ici. Les entendre et les lire m'a tout appris de la lecture et de l'écriture, au même titre que les plus grands de la littérature française à qui je dois tant.

Ce passage à la reconnaissance universelle de notre littérature québécoise et de nos grands écrivains, si l'Académie canadienne-française peut contribuer, vu son efficiente ouverture. à le favoriser, je suis heureuse et fière, par la minime reconnaissance individuelle qui méchoit aujourdhui, d'y participer. Disant ces choses, je madresse tout particulièrement à ceux des grands écri- 
vains dici dont jaime et admire les oeuvres et qui ne sont pas, ne feront sans doute jamais partie de cette assemblée. Cestavec ceux et celles mayant précédée ou maccompagnant dans le jardin des lettres québécoises que jentre à l'Académie. Leurs noms seront cités et leurs ouvrages, présents. Mes interventions, si précaires soient-elles, seront justiciables deux tous. Ici, je n'entre pas seule et cest pour ça que jy viens.

Dans le grand jardin des lettres dici, l'Académie est un petit jardin, à la fois public et intime. Nos prédécesseurs grecs avaient multiplié ces lieux de rencontres et lun n'excluait pas nécessairement lautre, les uns et les autres servant à la complexe et vitale insertion des littérateurs dans la cité, que ceux-ci soient poètes ou philosophes. Littéralement, l'AKADEMIKOS grec, c'est le Jardin; jardin public ou privé où, régulièrement, les collègues philosophes et poètes se retrouvaient pour discuter, discourir et parter.

Et je reviens au film de mes pensées du: début, délibérément abandonné en cours de route; après tout, le discours ne comportet-il pas lui aussi un cours libre de la pensée? Voici les séquences qui traversèrent mon esprit, entre l'invitation chaleureuse à venir faire partie de l'Académie et mon acceptation de celle-ci. Pendant toutes ces années où, pratiquant l'écriture, je ne pensais à peu près jamais à l'Académie, lorsque tout de même jy songeais quelque peu, ce sont tous les préjugés et toutes les images négatives rattachés à celle-ci qui meublaient mes pensées, comme sijavais été plus ou moins inconsciemment en accord avec une tradition littéraire qui fait de lacadémisme une réalité en tous points opposée à celle des dialogues aujardin. Sans trop réfléchir, ju voyais un style pompeux, des allures figées et quasiment un cimetière de la renommée. Celte chose que je ne connaissais pas, me semblait froide et stérile, remplie de vestons médaillés et de têtes couronnées qui ne créent plus et n inventent plus rien.

Puis, à réfléchir et à méditer, ce sont là dheureuses activités, fort utiles parfois, aux poètes comme aux autres, je réalisai lampleur de mes préjugés et de mon ignorance, je me demandai bien pourquoi, de l'histoire, je navais conservé de l'Académie que ses aspects académiques et pourquoi javais oublié, mais peut-être nai-je jamais vraiment su, en tout cas je navais plus jamais envisagé le côté jardin des choses, en cette matière. Vous dire, et même mavouer à moi-même les raisons de cette ignorance et de cette occultation, serait sans doute ennuyeux et surtout trop long pour ce présent discours. 
Quoi quïl en soit, pour lïnstant, je préfère fêter avec vous lavènement.

Quant à lignorance qui fut mienne, de mon village Amqui, nom amérindien qui signifie "là où les eaux samusent», de ce lieu extraordinaire doù je viens où parfois l'illisible devient aussi intelligent que le lisible (parmi ceux que jaimais, il $y$ en a qui ne savaient ni lire ni écrire, mais plusieurs possédaient une riche culture que jamais je ne renierai et pour eux tous, illettrés et lettrés qui mont enseigné l'amour de la connaissance, je témoignerai ici); de ce lieu où, à quatorze ans, je suis passée directement de la cour à bois et de mes rivières et du champ de vaches à l'Énéide de Virgile (dans le texte latin), à Hamlet ou Othello de Shakespeare (dans le texte anglais mais aussi dans les excellentes traductions de nos enseignantes, les savantes (Irsulines de Québec); de celieu Amqui, où, pour plusieurs, la transition s est faite très rapidement entre les connaissances sauvages et fantastiques d'un monde et lentrée dans le monde du savoir et des institutions; de cette vallée où, depuis des temps immémoriaux, tout voyage en littérature est une odyssée d'exil; de ce lieu, lon sait aussi, et cest là que la belle humilité est nécessaire, l'on sait d'un savoir profond et je dirais sans tracasseries, cette pensée que je résumerais par la phrase de Paul-Louis Courier. écrivain tourangeau du l8e siècle: "... la noblesse nest pas de rigueur pour entrer à l'Académie; l'ignorance, bien prouvée, suffit.»

En terminant, jaimerais dire mon contentement dentrer dans ce Jardin des dialogues en même temps que Marcel Dubé, écrivain pour qui jai toujours éprouvé de l'estime et de l'admiration. Je veux remercier tous les parents et amis qui sont venus ainsi que l'Académie et son Président Jean-Guy Pilon qui nous reçoivent aussi généreusement à cette fête que je souhaite la plus belle pour tous. Amusez-vous bien. 